\title{
Recent Results and Progress on Leptonic and Storage Ring EDM Searches
}

\author{
David Kawall \\ Department of Physics, University of Massachusetts Amherst, \\ Amherst, Massachusetts 01003, USA \\ kawall@physics.umass.edu \\ Published 29 February 2016
}

\begin{abstract}
The Standard Model is incomplete and unable to explain the matter-antimatter asymmetry in the universe. Many extensions of the Standard Model predict new particles and interactions with additional $C P$-violating phases that can explain this imbalance. Electric dipole moments (EDMs) of fundamental particles, which are generated by $C P$ violating interactions, can be enhanced by many orders of magnitude by contributions from this new physics to a magnitude within reach of current and planned experiments. New approaches to EDM searches using storage rings, and their sensitivity to new physics are presented.
\end{abstract}

Keywords: CP-violation; electric dipole moments; storage rings.

PACS numbers: 13.40.Em, 11.30.Er, 29.20.Dh.

\section{Introduction and Motivation}

Modern elementary particle physics faces a significant challenge when attempting to explain the observed matter-antimatter asymmetry in the universe. Sakharov showed that $C P$-violation is required to generate this baryon asymmetry. ${ }^{1}$ However, the only $C P$-violation observed, in $K$ and $B$ mesons, is parameterized in the Standard Model (SM) by a single phase in the CKM matrix and is insufficient by many orders of magnitude to explain the matter-antimatter asymmetry. New physics with new sources of $C P$-violation is required.

These new sources of $C P$-violation might be revealed in permanent electric dipole moments (EDMs) of fundamental particles. An EDM violates $T$ and $P$ symmetries, and by the $C P T$ theorem, $C P$. Since $C P$ and $P$ are violated in the $\mathrm{SM}$,

* On behalf of the Proton EDM Collaboration.

This is an Open Access article published by World Scientific Publishing Company. It is distributed under the terms of the Creative Commons Attribution 3.0 (CC-BY) License. Further distribution of this work is permitted, provided the original work is properly cited. 
radiative corrections incorporating $P$ - and $C P$-violating processes can generate a non-zero EDM from SM physics, but many orders of magnitude too small to be detected in current or planned experiments. ${ }^{2}$ An observation of a non-zero EDM entails the existence of new physics, and depending on the magnitude, may help explain the outstanding puzzle of baryogenesis.

An effective low energy MSSM CP-violating Lagrangian illustrates some of the contributions to leptonic and hadronic EDMs: ${ }^{3}$

$$
\begin{aligned}
\mathcal{L}_{\mathrm{eff}}= & \frac{g_{s}^{2}}{32 \pi^{2}} \bar{\Theta} G_{\mu \nu}^{a} \tilde{G}^{\mu \nu, a}+\frac{1}{3} w f^{a b c} G_{\mu \nu}^{a} \tilde{G}^{\nu \beta, b} G_{\beta}^{\mu, c}- \\
& \frac{i}{2} \sum_{i=e, \mu, u, d, s} d_{i} \bar{\Psi}_{i} \gamma_{5} \sigma^{\mu \nu} \Psi_{i} F_{\mu \nu}-\frac{i}{2} \sum_{i=u, d, s} d_{i}^{c} \bar{\Psi}_{i} g_{s} \gamma_{5} \sigma^{\mu \nu} \lambda^{a} \Psi_{i} G_{\mu \nu}^{a} .
\end{aligned}
$$

Here the $C P$-violating contributions to EDMs are: $\bar{\Theta}$, Weinberg 3-gluon term $w$, EDMs of $e$ and quarks $d_{i}$, and chromo-edms of quarks $d_{i}^{c}$. Current limits on the neutron EDM and ${ }^{199} \mathrm{Hg}$ limit $\bar{\Theta}<1 \times 10^{-10}$, where a priori $\bar{\Theta} \approx 0-2 \pi$. If Peccei-Quinn axions exist $\bar{\Theta} \rightarrow 0$, but no evidence for axions has been found. Radiative corrections to $\bar{\Theta}$ may induce non-zero EDMs.

For a given model of new physics, $w, d_{i}, d_{i}^{c}$ can be calculated. The $C P$-odd term $w$ cubic in $G_{\mu \nu}^{a}$ is not expected to dominate the EDM of a nucleon. To go from the quark level $\left(d_{u, d, s}, d_{u, d, s}^{c}\right)$ to nucleon level $\left(d_{n}, d_{p}\right)$ involves nuclear models. If $C P$-violation is due to $\bar{\theta}_{\mathrm{QCD}}$, we expect $d_{n} \approx-d_{p} \approx 3 \times 10^{-16} \bar{\theta} \mathrm{ecm}$. Conversely, if dominated by heavy quarks, we'd expect $d_{p} \approx d_{n}$. SUSY models predict $d_{n}$ and $d_{p}$ which depend on different linear combinations of quark and chromo-edms. In the case of $d_{e}$, it can be extracted from the EDM $d_{A}$, observed in the host atom or molecule, using $a b$ initio and semi-empirical techniques in atomic and molecular physics.

These considerations imply that EDM measurements in many systems $d_{e}, d_{\mu}, d_{p}, d_{n}, d_{d}, d_{3 \mathrm{He}}, \ldots$ are required to extract the parameters and hence identify the new sources of $C P$-violation. A single observation of an EDM, while of great importance, and definitive proof of new physics, must be accompanied by searches in complementary systems.

At present, all EDM search results are consistent with zero. The limit on the neutron $\mathrm{EDM}$ is $\left|d_{n}\right|<2.9 \times 10^{-26} \mathrm{ecm},{ }^{4}$ the electron EDM limit is $\left|d_{e}\right|<8.7 \times$ $10^{-29} \mathrm{ecm},{ }^{5}$ and the ${ }^{199} \mathrm{Hg}$ limit is $\left|d_{H g}\right|<3.1 \times 10^{-29} e \mathrm{~cm} .{ }^{6}$ The limit on the proton is inferred from the limit on ${ }^{199} \mathrm{Hg}$, and is $\left|d_{p}\right|<7.9 \times 10^{-25} \mathrm{ecm}$.

\section{Proton EDM Search in a Storage Ring}

As part of the worldwide effort, a storage-ring based experiment to search for the EDM of the proton is under development, with a target sensitivity of $10^{-29} \mathrm{ecm}$, representing nearly 3 orders of magnitude improvement, to probe new physics at the scale of $3000 \mathrm{TeV}$ for $C P$-violating phases of order $1 .^{7,8}$ 


\subsubsection{Experimental Technique}

For a particle in a storage ring with $\boldsymbol{\beta} \cdot \boldsymbol{E}=0$ and $\boldsymbol{\beta} \cdot \boldsymbol{B}=0$, the difference between the precession of the spin vector and momentum vector is $\boldsymbol{\omega}_{a}+\boldsymbol{\omega}_{\mathrm{EDM}}$ where:

$$
\begin{aligned}
\boldsymbol{\omega}_{a} & =\frac{e}{m}\left[a \boldsymbol{B}-\left(a-\frac{1}{\gamma^{2}-1}\right) \frac{\boldsymbol{\beta} \times \boldsymbol{E}}{c}\right], \\
\boldsymbol{\omega}_{\mathrm{EDM}} & =d(\boldsymbol{E}+\boldsymbol{\beta} c \times \boldsymbol{B}),
\end{aligned}
$$

where $a=(g-2) / 2$ is the anomalous magnetic moment of the particle, and $d$ is the EDM of the particle. ${ }^{9}$ In an all-electric ring, $\boldsymbol{B}=0$, and at the specific momentum where $\gamma=\sqrt{1+1 / a}, \boldsymbol{\omega}_{a}=0$ and $\boldsymbol{\omega}_{\mathrm{EDM}}=d \boldsymbol{E}$. For a proton, where $a_{p}=1.792847$, this magic momentum is $0.7007 \mathrm{GeV} / c$. In this case, the spin of stored longitudinally polarized protons would be frozen along the momentum vector, except for the effect of the storage ring radial electric field which would exert a torque on an non-zero proton $\mathrm{EDM} d_{p}$ causing the spin to precess out of the plane into the vertical, growing linearly with time.

The proposed storage ring is $500 \mathrm{~m}$ circumference, comprised of 40 sections of roughly $8 \mathrm{~m}$ long concentric cylindrical deflectors with $\approx 52 \mathrm{~m}$ bending radius and $3 \mathrm{~cm}$ spacing with $8 \mathrm{MV} / \mathrm{cm}$ radial electric field, separarated by 36 straight sections of $\approx 2.7 \mathrm{~m}$ length, and 4 long straight sections of $20.8 \mathrm{~m}$. Two of the long straight sections are for injection of polarized proton beams in opposite directions, the other two are for polarimeters. The shorter straight sections house SQUID magnetometers and electrostatic quadrupoles.

In a typical run, 100 bunches of $2.5 \times 10^{8}$ protons will be stored simultaneously in both the clockwise and counter-clockwise directions, with each bunch containing positive or negative helicity protons. Storage times are limited by the horizontal spin coherence time (maximized with feedback to an RF-cavity) to roughly $10^{3}$ seconds. An EDM of $10^{-29} e \mathrm{~cm}$ would lead to an early to late increase in the component of spin in the vertical of order a microradian. This is detected by scattering from a $6 \mathrm{~cm}$ long carbon target, where about $1 \%$ of the protons hit a detector after elastic spindependent pC scattering. A vertical component to the proton polarization yields a left-right asymmetry in the scattering. The systematic uncertainties in polarimetry have been investigated carefully at COSY-Jülich and should not limit the pEDM experiment. ${ }^{10}$

The statistical sensitivity is estimated as:

$$
\delta d_{p} \approx \frac{2 \hbar}{e E_{0} A P \sqrt{N_{c} f T_{\mathrm{tot}} \tau_{\mathrm{SCT}}}},
$$

where $E_{0}=8 \mathrm{MV} / \mathrm{m}$ is the radial electric field over $65 \%$ of ring, $A=0.6$ is the polarimeter analyzing power, $P=0.8$ is the proton beam polarization, $N_{C}=5 \times 10^{10}$ protons stored per cycle, $f=0.0055$ useful particle detected fraction in the polarimeters, $T_{\text {tot }}=10^{7} \mathrm{~s}$ running time per year, and $\tau_{\mathrm{SCT}}=10^{3}$ seconds spin coherence time, for a statistical uncertainty in one year of $4 \times 10^{-29} \mathrm{ecm}$. 
In some SUSY scenarios, at one-loop one expects:

$$
d_{p}=\left(10^{-22}-10^{-24}\right) \times\left(\frac{1 \mathrm{TeV}}{M_{\mathrm{SUSY}}}\right)^{2} \sin \phi e \cdot \mathrm{cm}
$$

so a limit of order $10^{-29} \mathrm{ecm}$ is probing new physics at the $3000 \mathrm{TeV}$ scale for $C P$-violating phases $\phi \approx 1 .^{11}$

\section{Proton EDM Systematics}

One of the most challenging systematics is from radial magnetic fields. A field of order $0.02 \mathrm{fT}$ would cause a precession of the proton spin into the vertical equivalent to the EDM limit sought. However, a radial field has the additional signature that it splits the CW and CCW beams vertically by $\delta y(\theta)$ around the ring:

$$
\delta y(\theta)=\sum_{N=0}^{\infty} \frac{\beta c R_{0} B_{r, N}}{E_{0}\left(Q_{y}^{2}-N^{2}\right)} \cos \left(N \theta+\phi_{N}\right),
$$

where $Q_{y}$ is the vertical tune, which will be modulated between 0.44 to 0.48 at roughly $10 \mathrm{kHz}$. This splitting, of order picometers for a $0.010 \mathrm{fT}$ radial field, can be detected with multiple SQUID magnetometers placed in the short straight sections of the ring. The splitting leads to a radial field produced by the beams at the vertical tune modulation frequency, large enough to be detected above ambient magnetic field and electronic noise with low- $\mathrm{T}_{C}$ SQUIDs, which have demonstrated sensitivities of $1 \mathrm{fT} / \sqrt{\mathrm{Hz}}{ }^{12}$ A schematic of the system is shown in Fig. 1.

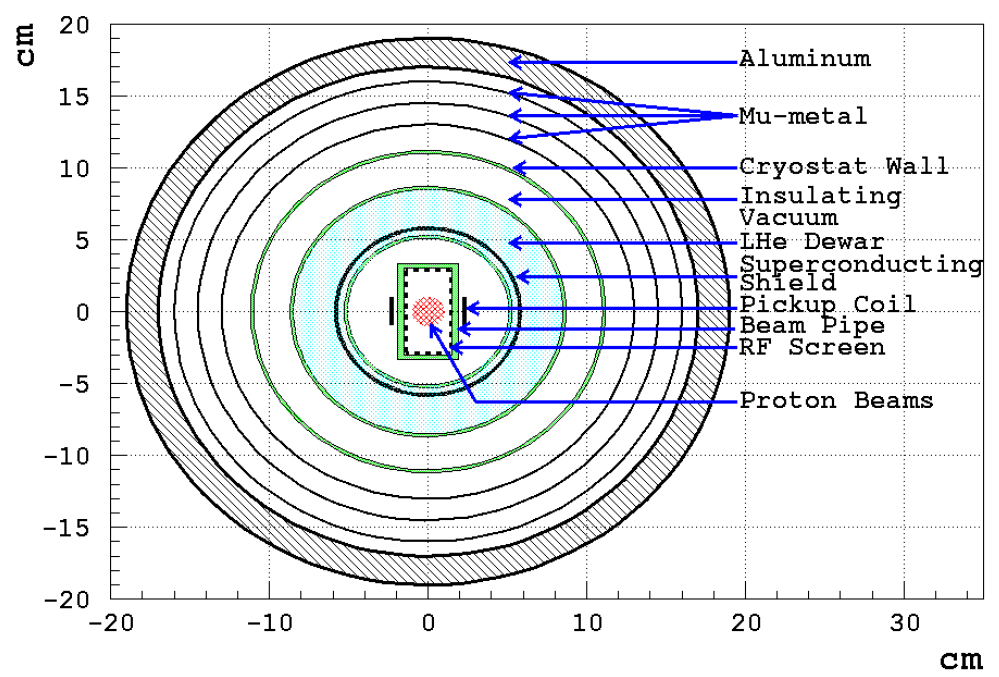

Fig. 1. A schematics illustrating the use of pickup coils connected to SQUIDs, in order to detect the vertical splitting of the counter-propagating proton beams caused by a radial magnetic field. 
Effects due to geometric phases can be limited by plate alignment, comparison of $\mathrm{CW}$ and CCW beams, and limiting the $B$ field. Other systematics are under control at the $10^{-29}$ e cm level. ${ }^{7,8}$

\section{Muon EDM Searches in a Storage Ring}

The current limit on the muon EDM is $\left|d_{\mu}\right|<1.8 \times 10^{-19} e \mathrm{~cm}$ at the $95 \%$ C.L. from the muon $g$-2 storage ring experiment at BNL. ${ }^{13}$ Naively, leptonic EDMs should scale as mass $m$, so the current electron EDM limit implies a muon EDM limit of order $d_{\mu} \approx \frac{m_{\mu}}{m_{e}} d_{e} \approx 1.8 \times 10^{-26} e \mathrm{~cm}$. It is important to check such scaling assumptions, and to search for EDMs in 2nd generation particles. The new muon $g$-2 storage ring experiment E989 at Fermilab should reduce the limits on the muon EDM by a factor of 100 to order few $\times 10^{-21} e \mathrm{~cm} .^{14}$

\section{Conclusions}

Techniques have been developed to conduct EDM searches sensitive to new physics at the scale of 1000's of TeV. A proposal to measure the EDM of the proton at the level of $10^{-29} \mathrm{e} \mathrm{cm}$ has been developed, and the upcoming new muon $g$-2 experiment at Fermilab should reduce the muon edm limits by another factor of 100 to order $10^{-21} e \mathrm{~cm}$.

\section{Acknowledgments}

Support from the US Department of Energy grant DE-FG02-88ER40415 is gratefully acknowledged.

\section{References}

1. A. Sakharov, JETP Letters 5, 24 (1967).

2. I.B. Khriplovich and S.K. Lamoreaux, CP Violation without Strangeness: Electric Dipole Moments of Particles, Atoms, and Molecules, (Springer-Verlag, Berlin, 1997).

3. D. Demir et al., Nucl. Phys. B 680, 339 (2004).

4. C.A. Baker et al., Phys. Rev. Lett. 97, 131801 (2006).

5. L. Baron et al. (ACME Collaboration), Science 343 (6168), 269 (2014).

6. W.C. Griffiths et al., Phys. Rev. Lett. 102, 101601 (2009).

7. V. Anastassopoulos et al. (Storage Ring EDM Collaboration), arXiv:1502.04317.

8. V. Anastassopoulos et al. (Storage Ring EDM Collaboration), A proposal to measure the proton electric dipole moment with $10^{29} e \cdot \mathrm{cm}$ sensitivity, http://www.bnl.gov/edm/.

9. I.B. Khriplovich, Phys. Lett. B 444, 98 (1998).

10. N.P.M. Brantjes et al., Nucl. Instrum. Meth. A 664.1, 49 (2012).

11. A. Czarnecki and W. Marciano in Lepton Dipole Moments, eds L. Roberts and W. Marciano (World Scientific, Singapore, 2010).

12. Y.H. Lee et al., IE-ICE Transactions on Electronics 88.2, 168 (2005).

13. G.W. Bennett et al. (BNL E821 Muon g-2 Collaboration), Phys. Rev. D 80, 052008 (2009).

14. J. Grange et al. (New Muon g-2 Collaboration), arXiv:1501.06858v1 (2015). 